\title{
A woman with cystic fibrosis, severe hypoxaemia, an atrial thrombus and a patent foramen ovale: a case report Nicholas J Simmonds ${ }^{1}$, Hilary Wyatt ${ }^{2}$, Raj Patel $^{3}$, Margaret E Hodson ${ }^{1}$ and Khin M Gyi ${ }^{1}$
}

\author{
Addresses: ${ }^{1}$ Department of Cystic Fibrosis, Royal Brompton Hospital and NHLI/Imperial College, Sydney Street, London, SW3 6NP, UK \\ ${ }^{2}$ Department of Cystic Fibrosis, King's College Hospital, Denmark Hill, London, SE5 9RS, UK \\ ${ }^{3}$ Department of Haematology, King's College Hospital, Denmark Hill, London, SE5 9RS, UK \\ Email: NJS* - nickjamessimmonds@yahoo.com; HW - hilary.wyatt@nhs.net; RP - raj.patel@kingsch.nhs.uk; MEH - m.hodson@imperial.ac.uk; \\ KMG - k.gyi@rbht.nhs.uk \\ * Corresponding author
}

Received: I4 September 2008 Accepted: I February 2009 Published: 20 July 2009

Journal of Medical Case Reports 2009, 3:8582 doi: 10.4076/1752-1947-3-8582

This article is available from: http://jmedicalcasereports.com/jmedicalcasereports/article/view/8582

(C) 2009 Simmonds et al; licensee Cases Network Ltd.

This is an Open Access article distributed under the terms of the Creative Commons Attribution License (http://creativecommons.org/licenses/by/3.0), which permits unrestricted use, distribution, and reproduction in any medium, provided the original work is properly cited.

\begin{abstract}
Introduction: Cystic fibrosis is usually associated with chronic pulmonary sepsis and frequent infective exacerbations. We report a very unusual cause of severe hypoxaemia in a woman with cystic fibrosis caused by thrombus formation in the right atrium.

Case presentation: A 2 l-year-old Caucasian woman with cystic fibrosis and a totally implantable venous access device presented with severe hypoxaemia. This was initially treated with antibiotics but her oxygen levels did not improve significantly. Subsequently, a transient ischaemic attack occurred. Further investigations, including a contrast echocardiogram and a cardiac magnetic resonance scan, revealed the presence of a large right atrial thrombus and right-to-left intracardiac shunt through a patent foramen ovale.

Conclusion: This case highlights the need to consider a right-to-left shunt in chronic respiratory diseases when hypoxaemia is out of proportion to the degree of lung function impairment. Totally implantable venous access devices should always be considered as a source of thrombus formation.
\end{abstract}

\section{Introduction}

Cystic fibrosis (CF) is an autosomal, recessively inherited disease caused by mutations in the cystic fibrosis transmembrane conductance regulator (CFTR) gene. It is common in Caucasian populations, giving rise to an incidence of approximately 1 in 2000 newborns. It has a predilection for the lungs and gastrointestinal tract, commonly manifesting as bronchiectasis and malabsorption secondary to pancreatic insufficiency. Progressive hypoxaemia occurs as a result of pulmonary sepsis and associated obstruction of the airways [1]. Despite being a chronic inflammatory condition with a purported increased incidence of thrombophilia [2,3], thromboembolic disease is rarely reported except in association with totally implantable venous access devices (TIVADs) [4-8] which are often inserted 
when frequent courses of intravenous antibiotics are required.

We describe a patient where a number of seemingly unrelated symptoms shared a mutual aetiology, presenting with severe hypoxaemia that was disproportionately low for her magnitude of pulmonary disease; investigations revealed a right-to-left intracardiac shunt as a result of a patent foramen ovale (PFO) and a right atrial thrombus.

\section{Case presentation}

A 21-year-old Caucasian woman with $\mathrm{CF}$, rheumatoid arthritis (RA) and a TIVAD presented with deterioration in her oxygen saturation, from a baseline of $95 \%$, to $88 \%$ in room air. This was attributed to an infective exacerbation as her forced expiratory volume in $1 \mathrm{~s}\left(\mathrm{FEV}_{1}\right)$ had decreased from 70 to $50 \%$ predicted. Arterial blood gas assessment revealed a $\mathrm{pO}_{2}$ of $5.8 \mathrm{kPa}$ and a $\mathrm{pCO}_{2}$ of $4.1 \mathrm{kPa}$ in room air. The diffusing capacity of carbon monoxide corrected for loss of alveolar volume (KCO) was $60 \%$ predicted. A thoracic computed tomography (CT) scan (including high resolution cuts and pulmonary angiography) revealed moderate bronchiectasis, small airways disease and mucus plugging with no evidence of pulmonary embolism. Her routine medications included nebulised preservative-free tobramycin (TOBI; Novartis, Basel, Switzerland), oral flucloxacillin, azithromycin, prednisolone (variable dose), multivitamins, vitamin E, calcium tablets, zoledronic acid, budesonide/formoterol inhaler, terbutaline inhaler, pancreatic enzymes, insulin glargine/lispro and naproxen. She was relatively immobile due to her RA and so did not complain of significant breathlessness. Intensive treatment with intravenous antibiotics and physiotherapy improved her $\mathrm{FEV}_{1}$ to baseline but her oxygen saturation only partially corrected ( $90 \%$ in room air).

One month later, she presented with transient expressive dysphasia and a right-sided hemiparesis. Oxygen saturation was $85 \%$ in room air and blood tests revealed the presence of lupus anticoagulants but anticardiolipin antibodies were negative (Table 1). Brain CT, lumbar puncture and carotid dopplers were all normal. The neurology resolved within 24 hours and a transient ischaemic attack (TIA) was diagnosed. A transthoracic echocardiogram revealed a right atrial mass (there was no evidence of intracardiac shunting or abnormal right heart pressures). Further evaluation with a contrast echocardiogram (Figure 1) and cardiac magnetic resonance imaging (Figure 2), confirmed the presence of a right atrial thrombus close to the distal end of her TIVAD and also a PFO with right-to-left shunting. A physiological shunt study was also performed, confirming a shunt fraction of $24 \%$. The patient was anticoagulated (international normalized ratio (INR) 2-3).
Table I. Laboratory results

\begin{tabular}{ll}
\hline FBC & Normal (including Hb \& plts) \\
U\&Es, LFTs & Normal \\
PT, APTT, Fib & Normal \\
Rheumatoid factor & Positive $(24 \mathrm{IU} / \mathrm{mL})$ \\
ANA & Positive \\
Lupus anticoagulants & Positive \\
Cardiolipin IgG/lgM & Negative \\
Antithrombin III & Negative \\
Protein C/Protein S & Normal \\
APC resistance & Normal \\
Prothrombin gene & Negative \\
\hline
\end{tabular}

Abbreviations: FBC, full blood count; U\&Es, urea and electrolytes; LFTs, liver function tests; PT, prothrombin time; APTT, activated partial thromboplastin time; Fib, fibrinogen; ANA, antinuclear antibody; Ig, immunoglobulin; APC, activated protein $\mathrm{C}$.

One year later, the patient was significantly better (oxygen saturation $95 \%$ in room air). Repeat echocardiography revealed resolution of the thrombus (Figure 3). Transcatheter closure of the PFO was performed but she remains anticoagulated due to the persistence of lupus anticoagulants and the TIVAD.

\section{Discussion}

Our patient had worsening hypoxaemia and a paradoxical thromboembolic event in the context of a TIVAD, a PFO and lupus anticoagulants. Initially, the hypoxaemia was attributed to an infective exacerbation, but other diagnoses were later considered as oxygen saturation did not improve with her $\mathrm{FEV}_{1}$. These included interstitial lung disease (in view of the RA) and pulmonary embolism (a rare diagnosis in CF with few reported cases - all in the presence of a TIVAD $[4,9])$. A PFO with right-to-left shunting was later confirmed, demonstrating that this is

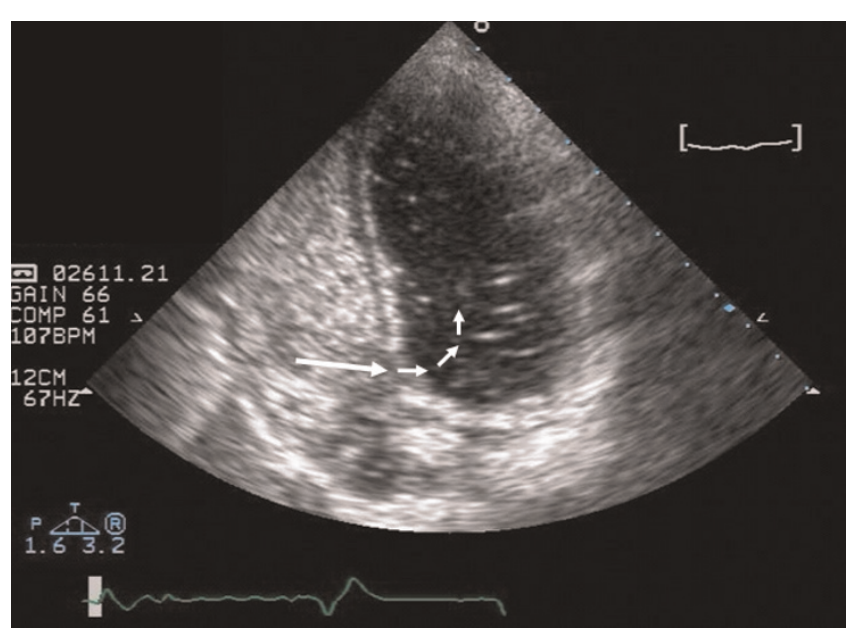

Figure I. Contrast ('bubble') transthoracic echocardiogram showing the presence of a patent foramen ovale (large arrow) with a right-to-left shunt (small arrows). 


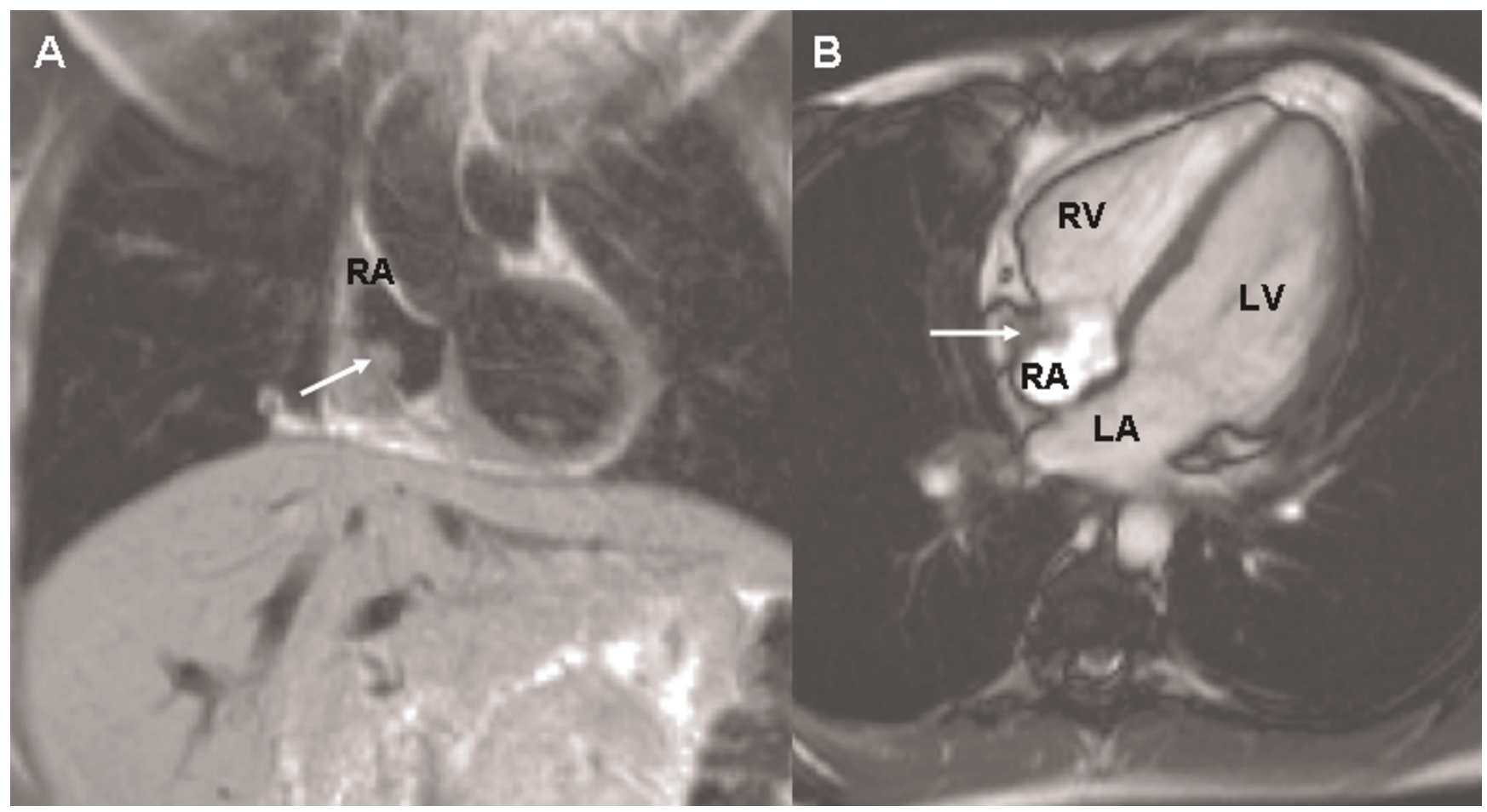

Figure 2. Cardiac magnetic resonance imaging showing the position of the thrombus (arrow) in coronal view (A) and transverse view (B). RV = right ventricle, $L V=$ left ventricle, $L A=$ left atrium and $R A=$ right atrium. Courtesy of $D r$ Raad Mohiaddin.

possible without significant pulmonary hypertension. We speculate this was a result of tricuspid valve obstruction from the thrombus [10]. Additionally, manoeuvres such as Valsalva and coughing are known to increase the shunt size, which are likely to have been important in this patient [11]. We know of no other association in the literature of thrombus formation and the persistence of a PFO. Despite resolution of the thrombus with anticoagulation, transcatheter closure of the PFO was still performed because her persistent procoagulant state was

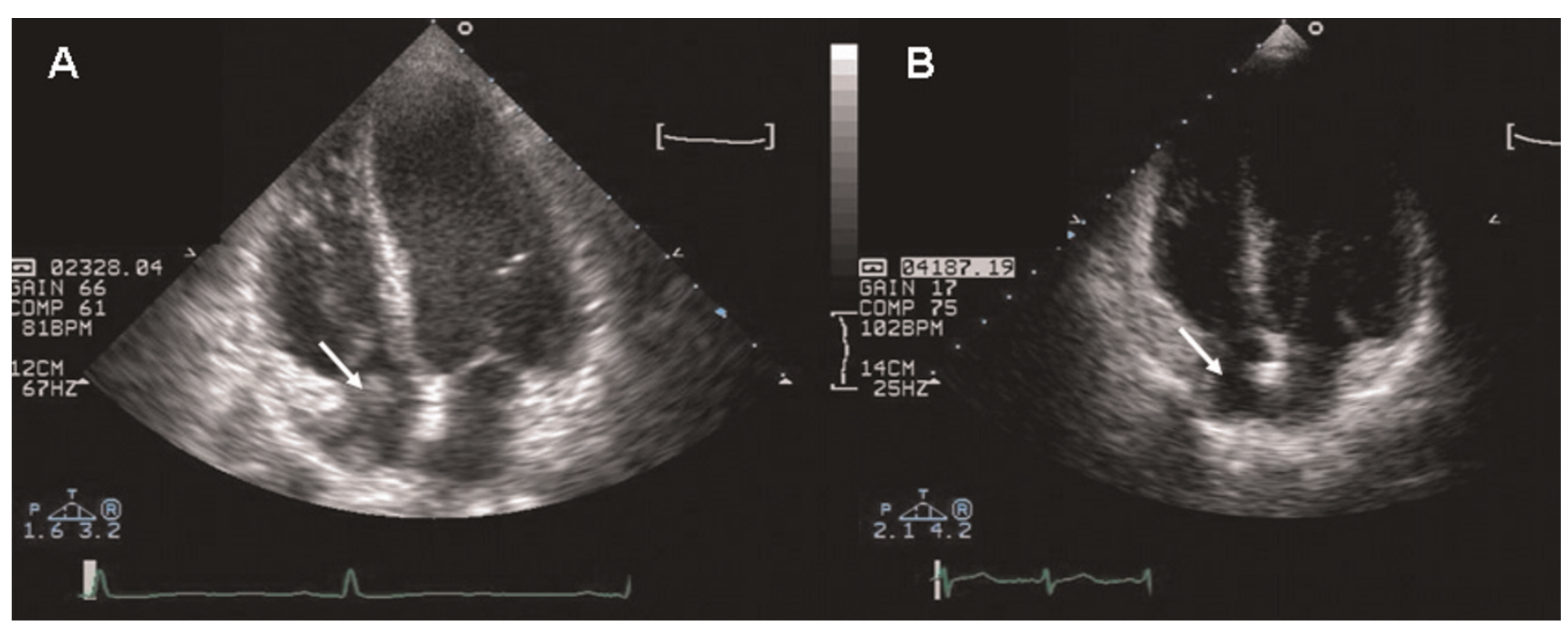

Figure 3. Four chamber view echocardiogram - pre (A) and post (B) treatment - showing resolution of the thrombus (arrow). 
considered to carry a significant risk of a further cerebrovascular event.

This was an unusual cause of hypoxaemia but should be considered when patients (especially with TIVADs) do not respond to conventional therapy. PFOs are not uncommon (incidence $27 \%$ in the general population [12]) and lupus anticoagulants are present in 5\% of the general population. Prior knowledge of her lupus anticoagulant status may have expedited the diagnosis but routine screening before TIVAD insertion is controversial as the presence of lupus anticoagulants do not predict a first thrombotic episode [13]. Furthermore, the efficacy of primary thromboprophylaxis for TIVADs is questionable; a recent consensus document advised against this in children as the available evidence does not show any benefit [14]. However, TIVADs are an independent risk factor for thrombosis (prevalence 5\% [15]) and therefore it is suggested in CF literature that thrombophilia screening should be considered [2].

\section{Conclusion}

This case highlights the need to be vigilant to other causes of hypoxaemia in chronic respiratory diseases when patients are disproportionately hypoxaemic in relation to their lung function. TIVADs should always be considered as potential sources of thrombosis and a right-to-left shunt needs to be carefully excluded when investigating hypoxaemia not explained by the more common sequelae of the underlying condition.

\section{Abbreviations}

$\mathrm{CF}$, cystic fibrosis; CFTR, cystic fibrosis transmembrane conductance regulator; CT, computed tomography; $\mathrm{FEV}_{1}$, forced expiratory volume in $1 \mathrm{~s}$; INR, international normalized ratio; KCO, diffusing capacity of carbon monoxide corrected for loss of alveolar volume; PFO, patent foramen ovale; RA, rheumatoid arthritis; TIA, transient ischaemic attack; TIVAD, totally implantable venous access device.

\section{Consent}

Written informed consent was obtained from the patient for publication of this case report and any accompanying images. A copy of the written consent is available for review by the Editor-in-Chief of this journal.

\section{Competing interests}

The authors declare that they have no competing interests.

\section{Authors' contributions}

NS collated the medical history and clinical investigations and also wrote the manuscript. HW, MH and KG were extensively involved in the management of the patient and made major contributors to writing the manuscript. RP provided expert haematological advice and contributed to the haematology aspects of the case report.

\section{Acknowledgements}

The authors acknowledge the Echocardiography and Cardiac Magnetic Resonance departments of the Royal Brompton Hospital for providing the images for this case report.

\section{References}

I. Davis PB: Cystic fibrosis since 1938. Am J Respir Crit Care Med 2006, 173:475-482.

2. Balfour-Lynn IM, Malbon K, Burman JF, Davidson SJ: Thrombophilia in children with cystic fibrosis. Pediatr Pulmonol 2005, 39:306-310.

3. Serisier DJ, Carroll MP: Catheter-related thrombosis associated with elevated factor VIII levels in cystic fibrosis. J Cyst Fibros 2006, 5:20I-204.

4. Yung B, Elborn JS, Campbell IA, Summers Y, Beckles M, Woodcock AA: Thromboembolism related to a Port-a-Cath device in a patient with cystic fibrosis. Thorax 1997, 52:98-99.

5. Playfor SD, Smyth AR: Paradoxical embolism in a boy with cystic fibrosis and a stroke. Thorax 1999, 54: I I39-1 I 40.

6. Espiritu JD, Kleinhenz ME: Paradoxical embolization in an adult patient with cystic fibrosis. Mayo Clin Proc 2000, 75: I I00-I I 02.

7. Barker M, Thoenes D, Dohmen H, Friedrichs F, Pfannenstiel C, Heimann G: Prevalence of thrombophilia and catheter-related thrombosis in cystic fibrosis. Pediatr Pulmonol 2005, 39:156-161.

8. Sritippayawan S, MacLaughlin EF, Woo MS: Acute neurological deficits in a young adult with cystic fibrosis. Pediatr Pulmonol 2003, 35: |47-|5|.

9. Sola JE, Stone MM, Wise B, Colombani PM: Atypical thrombotic and septic complications of totally implantable venous access devices in patients with cystic fibrosis. Pediatr Pulmonol 1992, 14:239-242.

10. Lee JJ, McKenzie FN, Novick R: Right atrial myxoma with cyanosis due to right-to-left shunting. Can J Cardiol 1990, 6:262264.

II. Clarke NR, Timperley J, Kelion AD, Banning AP: Transthoracic echocardiography using second harmonic imaging with Valsalva manoeuvre for the detection of right to left shunts. Eur J Echocardiogr 2004, 5:176-181.

12. Hagen PT, Scholz DG, Edwards WD: Incidence and size of patent foramen ovale during the first 10 decades of life: an autopsy study of 965 normal hearts. Mayo Clin Proc 1984, 59:17-20.

13. Walker ID, Greaves M, Preston FE: Investigation and management of heritable thrombophilia. Br J Haematol 200I, I I 4:5 I2528.

14. Monagle P, Chan A, Massicotte P, Chalmers E, Michelson AD: Antithrombotic therapy in children: The Seventh ACCP Conference on Antithrombotic and Thrombolytic Therapy. Chest 2004, I 26:645S-687S.

15. Munck A, Malbezin S, Bloch J, Gerardin M, Lebourgeois M, Derelle J, Bremont F, Sermet I, Munck MR, Navarro J: Follow-up of 452 totally implantable vascular devices in cystic fibrosis patients. Eur Respir J 2004, 23:430-434. 